\title{
Using the SimlnTech dynamic modeling environment to build and check the operation of automation systems
}

\author{
Albert A. Abalov ${ }^{1,}{ }^{*}$, Sergey V. Nosachev ${ }^{1}$, Viktor P. Zharov ${ }^{1}$ and Vsevolod A. Minko ${ }^{2}$ \\ ${ }^{1}$ Don State Technical University, 344000 Rostov-on-Don, Russia \\ ${ }^{2}$ Belgorod State Technological University named after V.G. Shukhov, 308012 Belgorod, Russia
}

\begin{abstract}
This article is devoted to the description of the simulation process of automatic control systems using the SimInTech dynamic modeling environment. Here is given a brief his-tory of the development of the software package from simulation to end-to-end process of creating the automatic control systems. Also describes the purpose of mathematical modeling of automatic control systems. The article presents basic information on modeling and development process in the environment. The example detailed step by step creation of an automation scheme is considered. The advantages and disadvantages of the SimInTech environment are shown in comparison with the competitive environment of Simulink.
\end{abstract}

\section{Introduction}

Engineering is intimately bound not only, immediately, with production (objects of management), but also an advancement of science and means of information processing in general. Thanks to larger opportunities of the modern computers, the sphere of activity and speed of realization of tasks of the modern engineers considerably increased. Besides, there were new tasks which each engineer has to be able to solve, for example, an integration of system of automatic control, change of parts of its model, simulation of interaction of separate elements and the whole system, simulation of mistakes, interactions of hardware and program parts, calculation of risks before realization of a product in actual life. New tools which list extended convenient means of calculations too are for this purpose used, there is a great many of programs capable to help to solve the particular circle of problems facing the engineer in this article more specifically will stop on Domestic development of SimInTech which strongly strengthened recently itself in the market and we will compare it to the main competitor in the market, very popular and used worldwide Matlab System Identification Toolbox and their Simulink package.

\footnotetext{
*Corresponding author: abal.26rus@yandex.ru
} 


\section{About SimlnTech}

SimInTech - the Russian system of the model focused projection of systems of automatic control (SAU).

The software of SimInTech consists of a graphical environment of development and the executive system of real time NordWind.

SimInTech - the environment of creation of mathematical models, control algorithms, interfaces of management and automatic oscillation of a code for programmable controllers and graphic displays.

NordWind - the executive system of real time which allows to start the created algorithms on the controller.

SimInTech is intended for a detailed research and the analysis of nonstationary processes in various objects of management.

SimInTech solves problems of model operation of objects of VPK/OPK, power objects, atomic objects, oil and gas objects, space objects, transport objects and others. Besides, SimInTech is intended for creation of exercise machines.

For creation of mathematical model of an object of SimInTech supports libraries for model operation: теплогидравлики / pneumatics; electrochains in operating and instantaneous values; power machines hydraulic/pneumatic; mechanical interactions; pointwise kinetics of neutrons; ballistics of spacecrafts; dynamics of flight of aircraft in the atmosphere; electric drives etc.

SimInTech allows to connect third-party program modules in various languages of programming ( $\mathrm{Si}$, Pascal, the Fortran and others).

For development of control algorithms in SimInTech there are all-technical libraries of blocks of automatic equipment which are turning on more than 300 blocks. Among them there are libraries: finite-state automations; relay automatic equipment; fuzzy logic.

Besides, SimInTech possesses: tools for creation of control interfaces; library of digital processing of signals; library of statistics; functional of optimization/selection of parameters; protocols of exchange (OPC, UDP, TCP/IP, MODBUS, RS, FMI etc);

the module for verification of the PLD code; the module of the analysis of reliability, safety and survivability of system on the key diagram.

The automatic generator of the SimInTech code for controllers allows to generate a code in languages:

ANSI C; ST;FIL.

In SimInTech there are templates allowing to make assembly of the executed code for various operating systems: QNX/KPDA; Linux;

\subsection{Advantages of SimlnTech}

- High flexibility of system;

- High speed of work of a mathematical core;

- High speed solutions to complex or mathematically stiff system of equations;

- The solution of heathydraulic problems at the level of concepts;

- Parallelization of calculations on the compute nodes with standard tools;

- The open interface for integration with various software ;

- Availability of tools for creating management interfaces;

- The ability to design projects for any template;

- Automatic generation of code configured for the target system and the possibility of flexible customization of code generation;

- Presence of execution environment for controllers. 
One of the main advantages to our Russian market, it is terminating finding of developers on the same territory with us, which multiplies both the speed and perception of one or another problem that has developed during the implementation of the system. Also in comparison with the competitor the package has more democratic price, which ultimately is possible due to the development on the territory of the Russian Federation, and not abroad.

\subsection{Disadvantages of Simintech}

Disadvantages are common to all tools of structural modeling. As in all such packages when creating complex models, it is necessary to build rather cumbersome multi-level block diagrams that do not reflect the natural structure of the modeled system. In part this general drawback of structural modeling packages is eliminated by using applications that implement the principle of physical multidomain modeling. Another problem arises here, namely, the insufficient capacity of the available data of the package in question, but also it is not possible to connect solutions to internal data in this moment, since this is realized in the main competitor MatLab Simulink.

\section{The description of MatLab Simulink as the competing platform}

Important advantages of the MATLAB package are openness, extensibility and its popularity, which allows finding solutions to a particular problem due to the fact that a huge number of users around the world use it. Most commands and functions of the system are implemented as text $\mathrm{m}$-files (with the extension . $\mathrm{m}$ ) and files in the $\mathrm{C}$ language, and all files are available for modification. The user is given the opportunity to create not only individual files, but also file libraries for the implementation of specific tasks.

The Simulink package is very convenient and natural for describing typical blocks of physical systems. The convenient interface reminds the designer, in which it is possible to quickly assemble a working model of any complexity with the help of various units - from the anti-block system of the car to the most complicated F-16 fighter. In this case, the user is given the opportunity to create their own blocks and combine existing ones into subsystems, which makes it possible to see a general structure that is not cluttered with secondary blocks.

The disadvantage of the package is connected with the desire to insert everything that is possible in the package. Overloaded with operators, commands, functions language, the main purpose of which is to improve visual perception. For example, the process of building electrical circuits in Simulink is frankly inconvenient - the ideology of the program does not allow connecting electrical wires in a natural way, forcing to resort to T-connectors, and sometimes to cumbersome tires. There is no more software platform, where MATLAB would be useful. The expensive software. If you are not a student, either be prepared to spend a substantial amount or cross the law by installing non-licensed software. And even if the student - the price is still more than decent. Low demand.

\section{Consideration of the implementation of a real task by means of SimlnTech}

\subsection{Creation of a thermal-hydraulic circuit file with a signal base}

Creating a new thermal-hydraulic circuit

To create a new thermal hydraulic circuit, you must perform the following actions:

1) In the main toolbar, select the "New Project" button.

2) Select the "Thermal hydraulics diagram" item in the drop-down menu (Figure 1) 


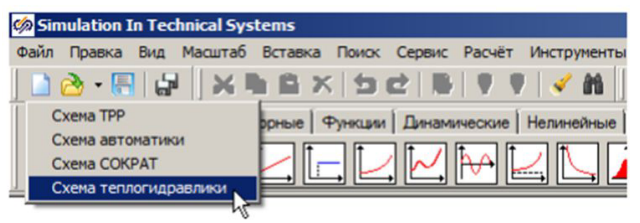

Fig. 1. Menu of creation a new project.

After this, a new schematic window will appear, in which a thermal hydraulics model will be created (Figure 2).

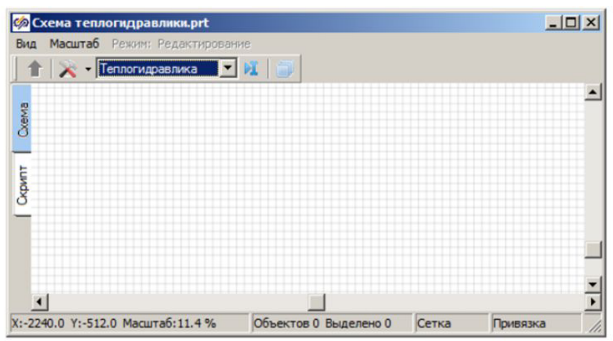

Fig. 2. Creating a schematic window to create a schematic of the thermal-hydraulic model.

For further work it is necessary to keep this scheme in the file with a new name in the same directory, in which the scheme of automatic equipment was kept. For this purpose it is necessary:

1) Select the "File" item in the main menu, select the "Save project" menu item in the drop-down list as...".

2) Using the standard file saving dialog, select the new name and directory to save. For example,"The scheme of thermal hydraulics.prt".

If necessary, the user can change the size and location of the window on the computer screen, using for this standard methods of working with windows.

\subsection{Database connection signals}

It is necessary for collaboration of several calculated codes that they used the same base of signals (which in our case is in earlier created signals.db file).

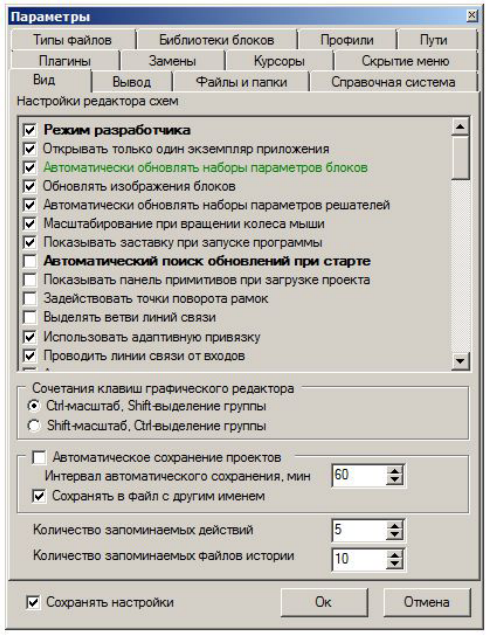

Fig. 3 Settings dialog box parameters of a program complex. 
To connect the database to the newly created thermal hydraulics project, it is necessary to translate the software package into the developer mode, for this you need to select the "File" item in the main menu of the program, then the "Parameters" sub-item. In the dialog box "Options" go to the "View" tab and check the "Developer mode" option (Figure 3).

Connection of the signal database to the thermal-hydraulic circuit is carried out as follows:

1) On the schematic window, click the "Calculation parameters" button:

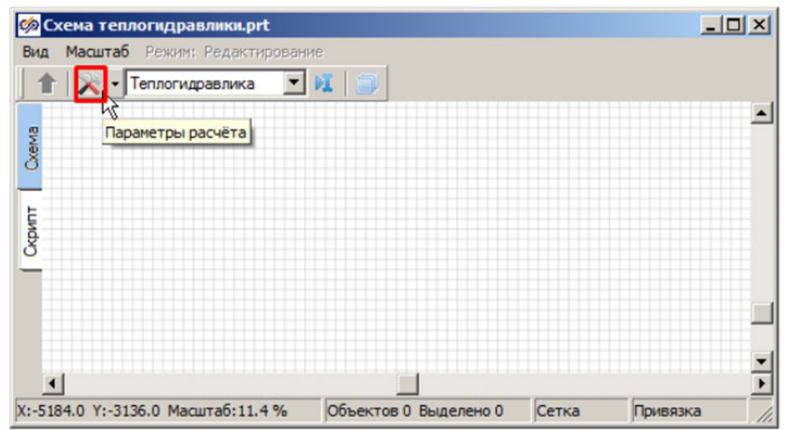

Fig. 4. Button access to the calculation parameters.

2) In the appeared dialog box of settings to go to the tab "Control" (Figure 5).

3) In a line of editing "The module of base of data of the project" it is necessary to enter the following text: "\$ Root $\backslash$ sdb.dll" (to enter without quotation marks; sdb.dll - a name of dynamic data of the program module of the database).

4) In the editing line "Project database name" enter an arbitrary file name to save the database. To use the database file of a previously created automation scheme, you must enter this file name (as in the first training task, ie in our case, "signals.db", Figure 5).

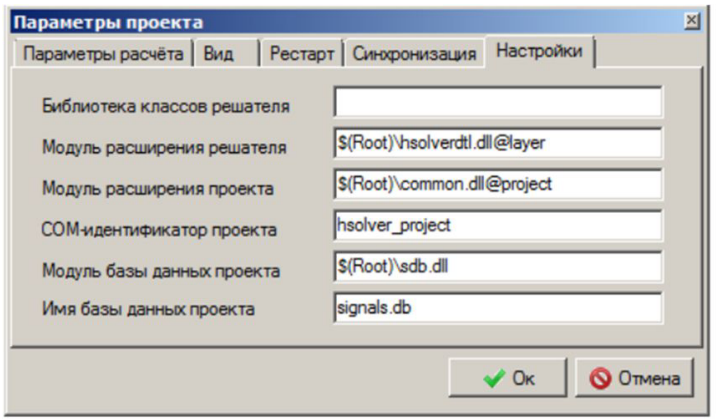

Fig. 5. Button access to the calculation parameters.

5) To close a dialog box pressing of the OK button (Figure 5).

\subsection{Connecting a schema to an existing database of signals}

To use the existing signaling database created when creating the automation scheme, you need to perform the following procedure:

1) Call the database editor through the main program menu. The menu item "Tools", the sub-item "Database ..." (Figure 6). 


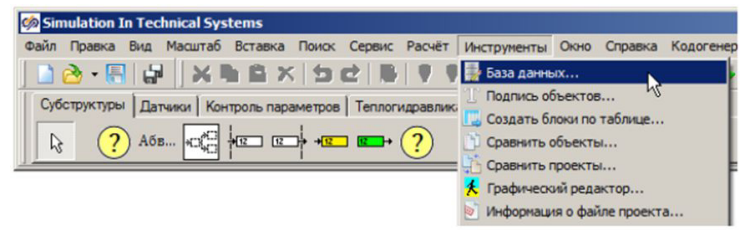

Fig. 6. The call database editor.

2) Since this scheme will be attached to an existing database, it is necessary to prohibit the automatic saving of the database when storing the thermal hydraulics circuit. Therefore, before closing the editor window, you need to make sure that the editor's settings do NOT assume automatic saving

Database. To do this, go to the "Settings" tab and uncheck "Save the database" (Figure 7) if there is a checkmark there.

3) Having disabled the option "Save Database", you must switch to the "Editor" tab and close the "Database Editor" dialog box by clicking on "Ok".

4) After that it is necessary to save the scheme and close the project.

These settings allow not to save the database while maintaining the thermal hydraulics scheme. This allows you to exclude erroneous changes to the database when editing the schema.

If all of the above actions were performed correctly, then after reopening the thermal hydraulic circuit, the signal database will automatically be loaded from the "signals.db" file and will contain all the signals generated when the automation scheme was created.

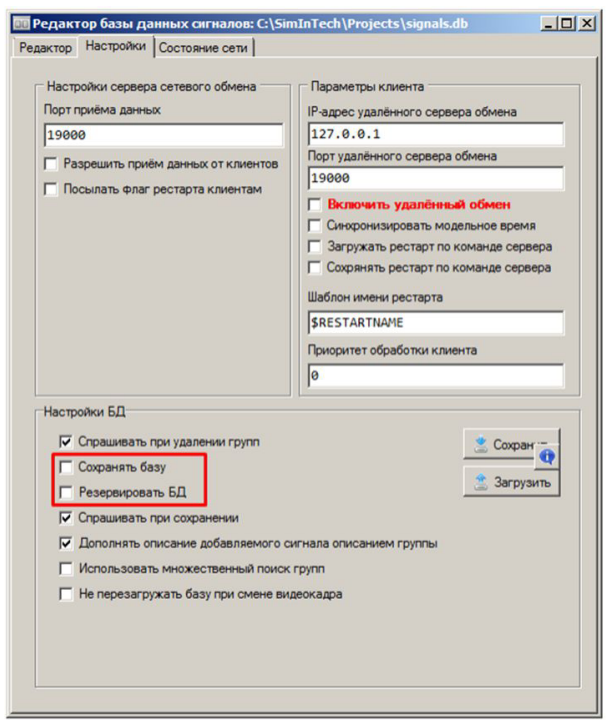

Fig. 7. Settings for saving a database for thermal-hydraulic schematic

Analyzing the main advantages and disadvantages of using the dynamic modeling environment of SimInTech, comparing it with the competitive environment of MatLab Simulink, we can conclude that the most suitable for our market is to see more the Domestic software product.

This is primarily as a material point of view the reporting package, and the maintenance and support of client base. Measurement by this method is most accurate and the measurement time in this case is minimal. 


\section{Conclusion}

Having analysed the main merits and demerits use of the environment of dynamic model operation of SimInTech, having compared it to the competitive MatLab Simulink environment, it is possible to draw a conclusion that the most suitable for our market is the Domestic software product. It is bound first of all both to the cost of the considered package, and from an upkeep, and support of client base.

\section{References}

1. I. A. Parshikov, etc. Humanities and Science University Journal, 5, 144 (2013)

2. B.A. Kartashov, etc. Environment of dynamic model operation of the technical SimInTech systems: Workshop on model operation of systems of automatic control (DMK Press Moscow, 2017)

3. V. V. Vasilyev, L. A. Simak, A. M. Rybnikova, Mathematical and computer model operation of processes and systems in the environment of MATLAB/SIMULINK (NAN of Ukraine, 2008)

4. A. P. Konovalchik, etc., New informational technologies in the automated systems, 20, $127(2017)$ 